\title{
Lavem as mãos, mas e o acesso à água? Prospecção tecnológica sobre PD\&I em Recursos Hídricos
}

Este trabalho apresenta um estudo de caráter categórico, sistemático e prospectivo que busca analisar o contexto atual da Pesquisa, Desenvolvimento e Inovação (PD\&I) na distribuição, acesso e conservação da água. Na primeira fase deste trabalho foi realizada uma busca dos descritores a serem utilizados, para isto foram utilizadas as bases de dados Scorpus e IEEE Xplore para selecionar os artigos científicos, e a base de dados Spacenet para selecionar as patentes relacionadas. A partir dos corpus textuais selecionados, os descritores foram categorizados através do software VOSviewer. Para a classificação da importância e da situação de semelhança dos trabalhos, foi utilizado o software StArt. Para a análise quantitativa dos corpus textuais utilizou-se o programa IRAMUTEQ. Considerou-se que os temas governança de tecnologias, gerenciamento de recursos, uso sustentável da água no âmbito familiar, estruturas de distribuição e coleta de águas para reuso e reservas subterrâneas, demonstram relevada importância no PD\&I para recursos hídricos. Este trabalho mostra ainda que é oportuno focar mais em estudos que visam desenvolver tecnologias industriais e métodos de governança para atender demandas de distribuição e reuso de água.

Palavras-chave: Planejamento governamental; Sustentabilidade; Gerenciamento; Higienização pessoal.

\section{Wash your hands, but what about access to water? Technological prospecting on RD\&I in Water Resources}

\begin{abstract}
A categorical, systematic and prospective study is presented, which seeks to analyze the current light on Research, Development and Innovation (RD\&I) in the distribution of access and conservation of water, thus helping organizations in their decision making, regarding the introduction of new technologies. directed to the management of water resources. The Scorpus and IEEE Xplore databases were used to build the article bank for the last 10 years, and the Patent2Net tool was used, which uses data from the Espacenet platform to generate a patent bank, outside the period of confidentiality of deposit, ie, 18 months. Also in this work, the VOSviewer software was used to categorize the main descriptors. For the classification of the importance and the similarity of the works, the software StArt was used and for the quantitative analysis of textual corpus, the software IRAMUTEQ was used. The five most recurrent classes were estimated in articles that worked with RD\&I on water resources, which are: (a) technology governance; (b) resource management; (c) sustainable use at home; (d) water distribution and collection structures for reuse and (e) underground reserves. This work also shows that it is opportune to focus more on studies that aim to develop industrial technologies and governance methods to meet demands for water distribution and reuse. In reference to patent texts, four classes of more recurrent terms emerged: (a) transport equipment, (b) sustainable use, (c) transport chain and (d) water treatment.
\end{abstract}

Keywords: Government planning; Sustainability; Management; Personal hygiene.

Topic: Conservação da Biodiversidade

Reviewed anonymously in the process of blind peer.
Received: 04/10/2020

Approved: $\mathbf{2 0 / 1 1 / 2 0 2 0}$
Luiz Diego Vidal Santos (1)

Universidade Federal de Sergipe, Brasil http://lattes.cnpq.br/7491112603328096 http://orcid.org/0000-0001-8659-8557 vidal.center@academico.ufs.br

Francisco Sandro Rodrigues Holanda Universidade Federal de Sergipe, Brasil http://lattes.cnpq.br/7935943969315043 http://orcid.org/0000-0003-3575-8105 fholanda@infonet.com.br

\section{Alceu Pedrotti (iD}

Universidade Federal de Sergipe, Brasil http://lattes.cnpq.br/5022174893387665 http://orcid.org/0000-0003-3086-8399 alceupedrotti@gmail.com

\author{
Catuxe Varjão de Santana Oliveira (iD \\ Instituto Federal de Sergipe, Brasil \\ http://lattes.cnpq.br/9781160589221829 \\ http://orcid.org/0000-0001-7276-4681 \\ catuxe.oliveira@academico.ifs.edu.br \\ Carlos Eduardo Celestino de Andrade (ib \\ Universidade Federal de Sergipe, Brasil \\ http://lattes.cnpq.br/6592469715706290 \\ http://orcid.org/0000-0001-9336-1072 \\ eng2carlos.eduardo@gmail.com \\ Arilmara Abade Bandeira (iD \\ Universidade Federal de Sergipe, Brasil \\ http://lattes.cnpq.br/9513043619290150 \\ http://orcid.org/0000-0001-6393-6242 \\ arilmara2015@gmail.com
}

Referencing this:

SANTOS, L. D. V.; HOLANDA, F. S. R.; PEDROTTI, A.; OLIVEIRA, C. V. S.; ANDRADE, C. E. C.; BANDEIRA, A. A.. Lavem as mãos, mas e o acesso à água? Prospecção tecnológica sobre PD\&l em Recursos Hídricos. Revista Ibero Americana de Ciências Ambientais, v.11, n.6, p.160177, 2020. DOI: http://doi.org/10.6008/CBPC2179-

$\underline{6858.2020 .006 .0015}$ 


\section{INTRODUÇÃO}

O uso dos recursos hídricos $(\mathrm{RH})$ de forma sustentável é objeto de ampla discussão na política mundial. Conforme reporte literário, os ambientes aquáticos lóticos e lênticos são aproveitados para distintas finalidades, tais como: produção de energia limpa, abastecimento de água, produção agrícola através da irrigação, produção aquícola ou para fins paisagísticos (EXTERCKOTER et al., 2015). O equilíbrio do meio ambiente é dependente de arranjos cíclicos e suscetíveis a ação do homem. Diversos estudos têm observado que existe uma forte correlação entre a ação humana e os impactos sobre os corpos hídricos usuais, tais como, a alteração na cadeia de ventos acarretando alteração de padrões de frequência de precipitação, alterações na vazão de rios provocando secas afetando os recursos naturais disponíveis (CHANAPATHI et al., 2019; DJEBOU, 2017; TERINK et al., 2010; XIE et al., 2020).

A água é um importante recurso ambiental, e após o uso se torna de difícil reconstituição por vias artificiais. Para o desenvolvimento sustentável de ambientes aquáticos, a distribuição e o desenvolvimento das pesquisas e inovações precisam ser expandidas para as demandas de sustentação dos recursos hídricos atuais, visto que a necessidade populacional da água aumenta substancialmente com o crescimento populacional (IWANAGA et al., 2020).

A agregação de dados resultantes de pesquisas é uma parte importante do processo de concepção do estado da arte, para o desenvolvimento de novas tecnologias voltadas a este recurso natural tão importante. Assim, o estudo sobre as proteções das patentes relacionadas ao contexto proposto e publicações científicas, apresenta grande importância, uma vez que o registro de novos produtos passíveis de patente é um fator importante para a mensuração da inovação (HOOCK et al., 2020), assim como as emergentes publicações em periódicos científicos reconhecidos.

A inovação aqui abordada pode ser definida como uma investigação, um descobrimento, um experimento, e adoção de novos processos, novos produtos ou novas políticas de governança. Ela resulta do relacionamento entre o meio e a necessidade, em que a aplicação do novo insight traz consequências econômicas para o local (WOLFFENBUTTEL et al., 2018). Pensando na perspectiva de RH, é possível que surjam inovações em diversos campos de exploração e conservação, solucionando diversas problemáticas da área.

A inovação social se relaciona diretamente ao contexto da sociedade, que emerge como o resultado da combinação entre uma função reparadora e a vontade de transformação social (MONTEIRO et al., 2019). Como forma de proteção aos possíveis direitos econômicos oriundos das produções intelectuais, torna-se desejável que essas inovações e tecnologias sejam depositadas, patenteadas e publicadas. Da mesma forma que pesquisas científicas, por questões éticas acadêmicas, devem ser publicadas a fim de que seja possível a propagação da informação em prol do desenvolvimento da comunidade acadêmica (LEITE et al., 2017; TONELLI et al., 2016).

Neste contexto, os diversos sistemas de propriedade intelectual incentivam a geração de novas tecnologias, produtos e processos, podendo resultar na criação de novos negócios em todas as áreas de 
atuação em RH, uma vez que permite ao autor/inventor explorar economicamente suas produções (HASAN et al., 2017). As patentes assim como as publicações científicas, representam instrumentos jurídicos diretos e indiretos de proteção do invento resultante de um esforço de pesquisa dos institutos de pesquisa, e de pesquisadores no desenvolvimento de processos tecnológicos e novos produtos. Isto torna um investimento seguro, rentável e legítimo se prevenindo do comportamento desleal que pode surgir da concorrência, evitando a comercialização de cópia não permitida, aspecto que desrespeita o esforço realizado pelos detentores originais e os gastos empregados no desenvolvimento (MARQUES et al., 2017).

Os documentos que compõem os pedidos de patente são constituídos por diferentes partes: relatório descritivo, reivindicações e resumo (INPI, 2011). Por sua natureza redacional, deve descrever o objetivo, funcionalidades e reivindicações das proteções solicitadas, se mostrando um texto capaz de demonstrar quais os aspectos da invenção e quais os pontos inéditos que trazem está novidade industrial, expressando desta forma possíveis inovações (WIPO, 2018). Tais documentos podem ser explorados por outros inventores e pesquisadores afim de compreender o estado da arte de determinadas pesquisas e áreas de invenções. Se mostra importante a busca da compreensão atual das propriedades intelectuais e inovações tecnológicas à luz das áreas derivadas dos contextos dos recursos hídricos, a fim de que seja possível compreender o contexto e caminho do desenvolvimento tecnológico.

Esta pesquisa vem propor uma metodologia de apoio à análise tecnológica através da abordagem sistemática e patentométrica, tendo como objetivo analisar o contexto atual sobre Pesquisa, Desenvolvimento e Inovação (PD\&I) na distribuição acesso e conservação da água. Neste contexto, a pesquisa busca auxiliar as organizações, inventores e pesquisadores na tomada de decisão no decorrer do desenvolvimento de novas tecnologias, assim como, identificar áreas de inovação e campos de aplicação de novas tecnologias direcionadas a toda a cadeia de uso da água.

Este artigo está organizado da seguinte forma: a Seção 1, aborda sobre a temática e objetivos pretendidos neste estudo, a Seção 2 apresenta a abordagem e ferramentas utilizadas para seleção, prospecção, classificação, análise dos textos e regras de associação para análise dos artigos de pesquisa e de patentes. Na Seção 3 é fornecida uma visão dos principais eixos e corpus dos quais derivam as principais classes encontradas nos resultados. 0 subtópico 3.1 aborda os resultados da prospecção e seleção dos textos a serem estudados, enquanto subtópico 3.2 trata sobre artigos de pesquisa. 0 subtópico 3.3 apresenta os resultados da análise fatorial dos artigos por correspondência e por fim, o subtópico 3.4 trata sobre textos patentários e seus resultados. Na Seção 4 são abordados os pontos concluintes deste estudo, seus principais alcances e futuras melhorias. E por fim, na Seção 5, são resumidos os principais resultados deste estudo, destacando as principais limitações e propondo recomendações para futuras pesquisas e experimentação do método proposto. 


\section{METODOLOGIA}

\section{Critérios de seleção sistemática dos trabalhos}

Como estratégia para busca da produção acadêmica a ser utilizada nesta rotina vinculada, foram construídos clusters utilizando a lógica baseada em descritores específicos - os descritores foram escritos na língua inglesa, vinculados por operadores booleanos (AND, OR e NOT), seguindo as regras descritas por Slamet et al. (2016), objetivando vincular as fontes de consulta com o tema proposto. Foram adotados como descritores primários os termos 'Water Resources' e 'Innovation'. Após definição destes descritores primários, a primeira execução foi realizada por dois pesquisadores, de forma independente e restrita. Para isso foi inicialmente utilizada como base principal de dados, o banco Scorpus, obedecendo rigorosamente os critérios de inclusão e exclusão, tais como: Apenas artigos completos, seguindo a série temporal de 10 anos (2010 a 2020), trabalhos escritos na língua inglesa, e a presença dos descritores primários nos seguintes campos: título (Title), resumo (Abstract) e palavras-chave (Keywords).

\section{Definição dos descritores secundários}

Para a investigação dos descritores secundários foi utilizado o software VOSviewer, destinado à análise de dados sistemáticos pelo método de clusterização de textos. O método consiste em um agrupamento textual, fazendo com que as palavras que façam mais sentido formem um número $\mathrm{k}$ de grupos, regidos por z centroides, em que, um centroide é um termo que reproduz o eixo central de um grupo, quando observado um determinado tema. Desta forma, foram selecionados os principais descritores agrupados a cada cluster observado.

\section{Patentometria}

Neste estudo foi utilizado o crawler Patent2net que consiste em um software multiplataforma opensource, que apresenta como principal funcionalidade a prospecção de informações na base de acomodação de dados Patentescope, pertencente ao European Patente Office (EPO) (FERRAZ et al., 2016). Ele extrai informações dos textos patentários contidos no diretório Espacenet. O Patent2net se mostrou eficiente, pois realiza buscas seguras e rápidas, e permite o uso de operadores lógicos e de identificadores de campo no diretório pesquisado. Dessa forma, o usuário pode realizar a busca utilizando os critérios de descritores existentes no título ou resumo das patentes, além da Classificação IPC descrita na patente, do inventor da propriedade industrial, do País de propriedade unionista, do ano de publicação, entre outros.

Os procedimentos de busca desta pesquisa seguiram os mesmos descritos por (FERRAZ et al., 2016). Foram utilizados os mesmos descritores utilizados na busca dos artigos de pesquisa.

\section{Seleção por similitude e contexto dos documentos analisáveis}

Buscando fornecer os meios pelas quais as melhores evidências atuais da pesquisa possam ser integradas ao real estado do desenvolvimento tecnológico na área de recursos hídricos, foi adotado neste 
trabalho, durante a fase de disposição categórica de qualidade e relevância dos estudos como o tema, o protocolo baseado nas orientações de Kitchenham et al. (2009). Essas orientações definem que o protocolo de análise por abordagem sistemática deve ser um modo de avaliar, identificar e explicar os estudos proeminentes para responder uma questão ou um fenômeno, referente a uma determinada temática, e que deve ser composto por três fases: Planejamento, Execução e Publicação dos Resultados (KITCHENHAM, 2004).

O protocolo consiste na seleção de documentos, tomando como pressupostos os artigos que apresentem melhor pontuação por score de qualidade, e cumpram os dois critérios de aceitação, provenientes da fase de planejamento (KITCHENHAM et al., 2009). Esse trabalho adotou como primeiro critério de classificação, a Estratégia de Seleção Automática de Citação em Pontuação (Score Citation Automatic Selection Strategy-SCAS), que consiste em categorizar os artigos por número de ocorrência da repetição de citações observadas automaticamente (sistema), e visualmente (leitor), entre os estudos pesquisados para apoiar a seleção inicial de estudos (triagem inicial) (OCTAVIANO et al., 2015).

Como segundo bloco de critérios para aceitação: trabalhos que apresentam os dois descritores primários, apresentassem relação entre os descritores primários e os secundários, e que tenham similaridade entre as referências citadas. Como critério de classificação para pontuação de score foi assumido que, para cada descritor primário e secundário que estivesse presente no título, seria contabilizado 20 pontos, e os que estivessem no resumo e palavras-chave receberiam 5 pontos.

Ressalta-se que, no contexto deste trabalho, o termo triagem inicial é usado para referir à atividade de selecionar artigos relevantes para o cenário de pesquisa, após a busca direcionada, utilizando os descritores primários e secundários. Como ferramenta de categorização, foi utilizado o software StArt (State of the Art through Systematic Review) (LAPES, 2005).

\section{Análise quantitativa de dados textuais}

Após a seleção dos trabalhos diretamente relacionados com o tema proposto desta prospecção tecnológica, baseados nas temáticas emergidas dos descritores primários e secundários, os quais cumpriram as regras supracitadas para a seleção inicial, foram realizadas lexicometrias e análises de discursos nos textos presentes. O software IRAMUTEQ (MARCHAND et al., 2012) foi utilizado nesta fase. Este software utiliza Unidades de Contexto Iniciais (UCls) na construção do modelo para análise. Ao se trabalhar com artigos científicos, cada conjunto de texto deve compor uma UCI. Um conjunto de UCl é conhecido como corpus de análise, os quais o software segmenta em textos de aproximadamente três linhas, chamados de Seguimento de Texto (ST). Após a segmentação dos textos, o software realiza uma Categorização Hierárquica Descendente (CHD) dando origem a classes lexicais caracterizadas pelo vocabulário e pôr em segmentos de textos que partilham o mesmo vocábulo (FERNANDES et al., 2017).

Neste sentido, diferentes classes resultantes representam o espaço de sentido das palavras narradas, surgindo, assim, elementos pertencentes aos temas observáveis no tema proposto (CAMARGO et al., 2013; FERNANDES, 2016; GAVASSO et al., 2016). 
Foram consideradas como palavras analisáveis, os termos caracterizados como substantivos, adjetivos e verbos. Demais conjuntos e termos presentes nos STs foram omitidos da CHD. Na construção e análise dos dados, foram utilizados os softwares Freeplane e o Carrot2, tanto para artigos quanto para textos patentários. Eles usam mecanismos de renderização 3D para exibir grandes redes em tempo real e acelerar a exploração (BASTIAN et al., 2009). Para a elaboração do corpus foi assumida a premissa de que, cada artigo e textos patentários correspondem a uma $\mathrm{UCl}$, em que, cada três linhas de texto presentes no resumo dos trabalhos correspondem a um ST.

\section{Qualidade metodológica}

A avaliação da metodologia proposta neste estudo foi mediada pela técnica desenvolvida por Costa et al. (2015), tendo como base a Assessment of Multiple Systematic Reviews (AMSTAR) proposta por Shea et al. (2007). Para tal, foi aplicado um checklist contendo 14 perguntas, que buscam verificar o nível de significância e inferência científica dos dados coletados e organização da revisão, vide (COSTA et al., 2015).

A Figura 1 apresenta a estratégia de busca, exclusão e seleção sistemática dos artigos de pesquisa e textos patentários adotada nesta pesquisa.

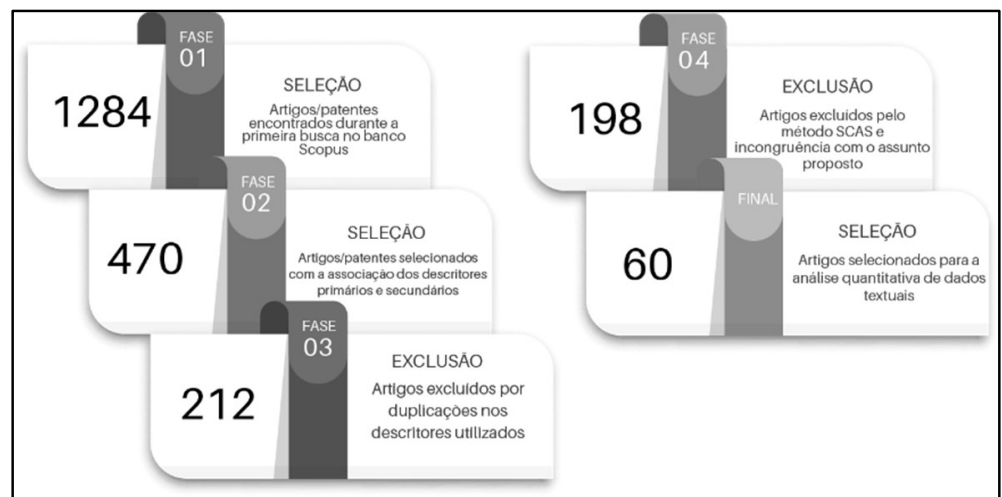

Figura 1: Fluxograma de abordagem de seleção, categorização e distribuição dos artigos encontrados relacionados as áreas de pesquisa sobre recursos hídricos. No lado esquerdo da figura tem-se o número de artigos/patentes selecionados e ao lado direito a fase correspondente.

\section{RESULTADOS}

\section{Resultados bibliográficos}

Inicialmente a recuperação bibliográfica através do Scopus e do IEEE Xplore resultou em 1284 artigos publicados entre 2010 e 2020, os quais, após utilização do VOSviewer, resultaram 10.914 descritores, divididos em 02 (dois) clusters: Recursos Hídricos (RH) e Inovação (In). Conforme a nuvem apresentada na Figura 2, ficou evidente a interação entre os trabalhos e os descritores, onde foi possível identificar a integração dos dois clusters, tendo o mínimo de 10 ocorrências, e acima de 100 interações dentro dos clusters, resultando ao final 470 artigos selecionados.

A Tabela 1 agrupa os dois principais clusters, Recursos Hídricos (RH) e Inovação (In), utilizados para realização da seleção direcional mais profunda relativa ao enfoque proposto. A análise da Tabela 1 se mostra relevante quando se observa os descritores secundários resultantes, interacionados com os dois descritores 
primários e áreas de PD\&I em recursos hídricos mais citadas no primeiro corpus. Os descritores são: gerenciamento hídrico, desenvolvimento sustentável e abastecimento de água, eles alcançam maiores quantidades de ocorrências em artigos e textos patentários, ou seja, aparecem 249, 197 e 160 vezes em textos diferentes, respectivamente.

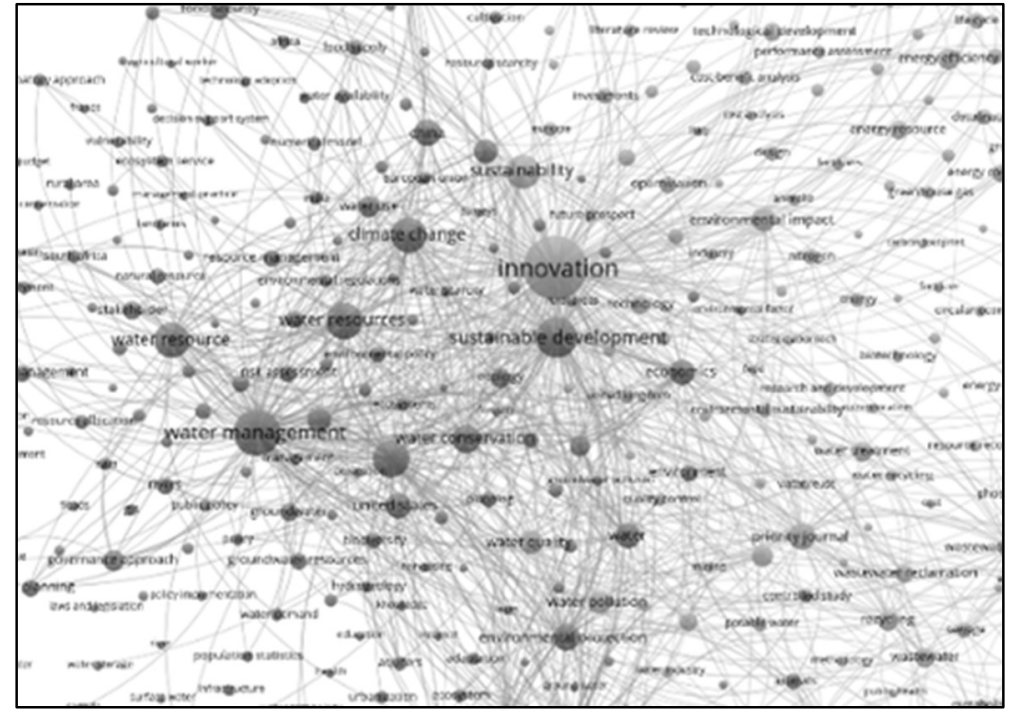

Figura 2: Síntese da rede de descritores objetivado na busca dos principais temas relacionados à inovação e aos recursos hídricos.

Desta forma, as percepções de relevância destes temas mostram, ao que parece uma visão parcial de que, o uso destes descritores secundários será mais fiel ao objetivo da prospecção aqui proposta. Haja vista que, todos os descritores elencados estão intrinsecamente ligados entre si quando se observa o tema geral - RH (CHANAPATHI et al., 2019; DJEBOU, 2017; TERINK et al., 2010; XIE et al., 2020).

Tabela 1: Principais clusters obtidos após busca no banco de dados Scopus utilizando os descritores water resources e innovation.

\begin{tabular}{|c|c|c|c|}
\hline Clusters & $\begin{array}{l}\text { Descritor } \\
\text { observado }\end{array}$ & $\begin{array}{l}\text { Quantidade de ocorrências nos em } \\
\text { artigos }\end{array}$ & $\begin{array}{l}\text { Força total das ligações entre os } \\
\text { descritores }\end{array}$ \\
\hline $\begin{array}{l}\text { Cluster } \\
\mathrm{RH}\end{array}$ & Gerenciamento hídrico & 249 & 2844 \\
\hline Cluster In & Desenvolvimento sustentável & 197 & 2160 \\
\hline $\begin{array}{l}\text { Cluster } \\
\mathrm{RH}\end{array}$ & Abastecimento de água & 160 & 2076 \\
\hline $\begin{array}{l}\text { Cluster } \\
\mathrm{RH}\end{array}$ & Alteração climática & 143 & 1514 \\
\hline Cluster In & Sustentabilidade & 130 & 1499 \\
\hline $\begin{array}{l}\text { Cluster } \\
\mathrm{RH}\end{array}$ & Conservação da água & 129 & 1364 \\
\hline
\end{tabular}

A Tabela 2 apresenta a combinação dos descritores primários e secundários nas páginas de cobertura ou títulos, obtidos a partir dos dados minerados nos sites Scopus e IEEE Xplore, durante o período de análise - março de 2020. A partir dos descritores primários e os seis secundários, foram excluídos 1026 trabalhos encontrados nesta fase. O corpus elaborado para análise da dimensão do desenvolvimento de inovações e empreendedorismo, contemplando as áreas de estudos ligadas aos recursos hídricos se deu pelo levantamento de 258 artigos distintos (Tabela 2). 
Tabela 2: Resultados da seleção de artigos e descritores primários e secundários no banco de dados Scopus entre 2010 2020.

\begin{tabular}{ll}
\hline Combinações de descritores na página de cobertura ou título & N. Artigos não repetidos \\
\hline ("water resources" AND innovation AND "water management") & 68 \\
("water resources" AND innovation AND “sustainable development") & 54 \\
("water resources" AND innovation AND “climate change") & 47 \\
("water resources" AND innovation AND "water supply") & 37 \\
("water resources" AND innovation AND sustainability) & 29 \\
("water resources" AND innovation AND "water conservation") & 23 \\
\hline Total & $\mathbf{2 5 8}$ \\
\hline
\end{tabular}

Observa-se na Tabela 3 a seleção de contagem de trabalhos utilizando o protocolo recomendado por Kitchenham et al. (2009), assim como, a regra de seleção por estratégia automática de citação em pontuação. É possível observar na Tabela 3 um total de 60 artigos, oriundos dos 258 trabalhos obtidos na fase anterior. Tais trabalhos apresentaram valores de pontos de escores descritos na Tabela 3.

Tabela 3: Quantidade de trabalhos selecionados por nível de scores alcançados por pontos predeterminados na fase de seleção de similitude e contexto.

\begin{tabular}{ll}
\hline Contagem de pontos (score) & Quantidade de artigos \\
\hline$>=75$ & 4 \\
Entre 74 e 60 & 6 \\
Entre 59 e 45 & 4 \\
Entre 44 e 30 & 27 \\
Entre 29 a 15 & 19 \\
\hline Total & $\mathbf{6 0}$ \\
\hline
\end{tabular}

Importante salientar que os demais trabalhos excluídos não cumpriam os requisitos requeridos pela classificação 'Estratégia de Seleção Automática de Citação em Pontuação', assim como não apresentavam pontos acima de 15, requisito mínimo para classificação por score, tais como: relevância do tema, quantidade de descritores citados no título, abstract, palavras-chave e um nível aceitável de similitude dos principais artigos analisados. Desta forma, os 198 artigos foram excluídos do corpus final.

\section{Análise lexical dos textos}

O uso do programa IRAMUTEQ trouxe a vantagem de organizar, codificar, e categorizar as informações, o que proporcionou a localização ágil de todo o corpus utilizado na pesquisa qualitativa dos artigos e patentes estudados nesta pesquisa. Para o alcance dos resultados, buscando realizar a CHD o software percorre três etapas: a codificação e a preparação textual inicial, a CDH assim como a interpretação das classes finais (SOUZA et al., 2018).

Buscando construir todos os conjuntos de textos necessários ao desenvolvimento do corpus de análise, a preparação inicial dos textos se deu com a inclusão em um único corpus, os resumos, títulos e palavras-chaves de cada documento selecionado. Assim, dos 60 artigos resultantes, surgiram $60 \mathrm{UCI}$ organizados em um único arquivo, em que, cada $\mathrm{UCl}$ foi apartada por uma linha contendo somente uma variável (n), descrita conforme um número dado a cada artigo estudado, (*****TEXTO_n). O corpus organizado foi salvo como arquivo de texto (formato.txt), utilizando caracteres no padrão UTF-8 (Unicode Transformation Format 8bit codeunits), utilizando o software Notepad++. Foram suprimidos todos os caracteres especiais contidos no texto, $(* \% \& \wedge)$, foi realizada também a amarração de palavras compostas, 
como por exemplo: 'water_resources', evitando que o software as identificasse como palavras distintas.

A pesquisa resultou em um corpus de artigos contendo $60 \mathrm{UCl}$, originando $448 \mathrm{STs}$, os quais continham 24291 ocorrências (Palavras, Formas ou Vocábulos), 2598 palavras distintas e 1348 hapax (palavras com uma única ocorrência). Exibindo uma frequência de 9,31\% para ocorrência de palavras, e uma frequência média de $48,75 \%$ de ocorrência por ST. O programa utiliza para a criação da matriz analítica de palavras, o teste estatístico qui-quadrado $\left(\chi^{2}\right)$, o qual demonstra a força associativa entre as palavras contidas em cada cluster resultante (CAMARGO et al., 2013). Uma boa força associativa é considerada quando o $\chi^{2}$ apresenta valores acima de 3,84, representando $p$-value $<0,0001$, (SOUZA et al., 2018). Um valor inferior aos 3,84 demonstram pouca correlação entre as variáveis. As classes resultantes desta pesquisa pós CHD podem ser observadas na Figura 3.

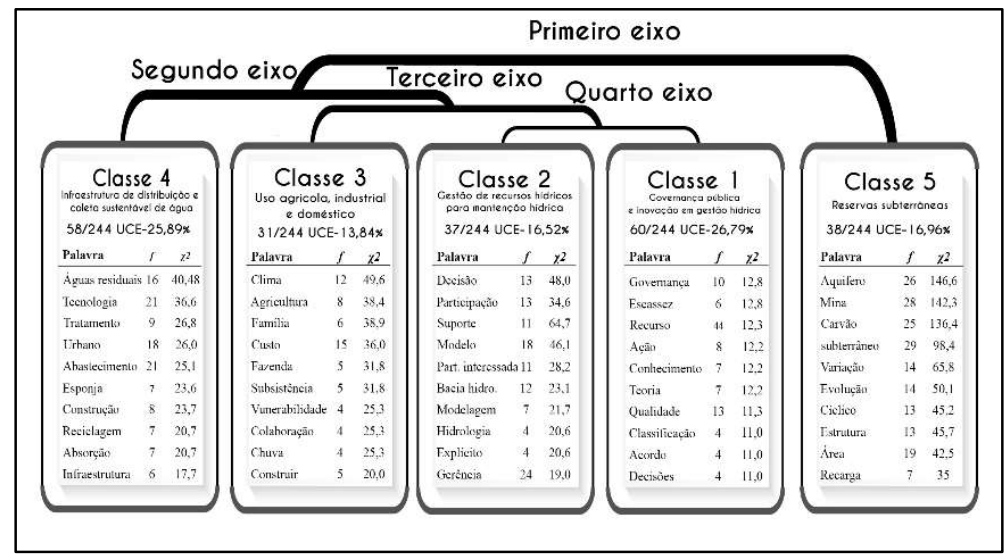

Figura 3: Dendrograma representativo dos STs observadas nos artigos de pesquisa.

* Classes com grau de significância estatística com a classe de $<0,0001$.

Após redução dos vocábulos as suas raízes surgiram a partir de 2958 lemas, resultando em 448 STs classificados, sendo aproveitado por fim 341 STs, uma taxa de retenção de 76,12 \% do corpus, satisfazendo os critérios sugeridos pela literatura pertinente. Emergiram 2786 formas ou palavras ativas analisáveis e 165 formas suplementares. Ainda na Figura 3, é possível observar a Classificação Hierárquica Descendente (CHD) formada. Pela leitura das palavras em que apresentaram os maiores valores de qui-quadrado como também os seguimentos de textos característicos com maior pontuação de score, por soma absoluta dos $\left(\chi^{2}\right)$ das palavras mais destacadas nos seguimentos, foi possível alcançar os objetivos da pesquisa primária, voltada a verificar as atuais áreas de pesquisa desenvolvimento e inovações na área de recursos hídricos.

Desses dois grandes eixos temáticos resultam 5 classes lexicais caracterizadas pelo vocabulário e por segmentos de textos que compartilham o mesmo vocabulário. O primeiro eixo foi desmembrado em dois subcorpus. Na primeira classificação do primeiro eixo está presente isoladamente a Classe 5, com 38 STs, correspondendo a $16,96 \%$ do total e os demais subcorpus subsequentes.

Já o segundo subcorpus (segundo eixo) houve uma nova subdivisão: mais dois subcorpus, que engloba a Classe 4 com 58 STs, que representa $25,89 \%$ do total e o terceiro eixo com mais uma subdivisão. Uma terceira subdivisão (quarto eixo) contendo as Classes 1 e 2 com 60 STs (26,79\%) e 37 STs, 16,52\% do total respectivamente. Ainda neste eixo, surgiu a Classe 3 com 31 STs representando 13,84\% do total. Para definição do perfil de áreas descritas nesta pesquisa, foram selecionados os termos que apresentaram maior 
grau quantitativo de significância baseada na análise do qui-quadrado $\left(\chi^{2}\right)$.

O primeiro grande eixo é composto por uma classe que contempla artigos relacionados a aquíferos e ambientes subterrâneos e outros subcorpus que tratam sobre governança e inovação em recursos hídricos. Do segundo eixo emerge um subcorpus e o terceiro eixo. O subcorpus deste eixo contempla a classe 4 que trata sobre infraestrutura, método de distribuição de água potável e coleta de água residual em ambientes urbanos. Os subcorpus deste eixo contemplam separadamente a classe 3 , que aborda o uso doméstico e padrões de avaliação de qualidade, e as classes 2 e 1 que tratam de gestão e governança de pessoas e recursos hídricos respectivamente, Figura 3.

\section{Classe 1: Governança pública e inovação em recursos hídricos}

A classe 1 que trata sobre governança pública e inovação em gestão hídrica, presente em grande parte dos artigos publicados no ano de 2013 , corresponde a $26,79 \%$ das UCEs no corpus. Os seguintes segmentos de textos descrevem melhor o conteúdo da classe: "O sistema socioeconômico consome recursos hídricos quando produz serviços e poluentes"(REN et al., 2016). "Este artigo examina a extensa e evolutiva literatura relativa à economia da política da água que oferece aos políticos uma forma de pensar e um conjunto de competências" (CONVERY, 2013); "Examinar o papel da comunicação no processo que orienta os agentes públicos a integrar as obrigações morais implícitas pela adoção de princípios de sustentabilidade nas suas escolhas de ação e a reexaminar as suas práticas" (DEL CORSO et al., 2015).

\section{Classe 2: Gestão de recursos humanos para manutenção hídrica}

A segunda classe, pertencente ao quarto eixo, corresponde a $16,72 \%$ do corpus estudado e publicados em sua maioria no ano de 2014, é composta por artigos que tratam sobre gerência de recursos humanos para manutenção das fontes hídricas. Alguns segmentos de textos ilustram os conteúdos pertencentes a classe: "Foi utilizado o método para medir a eficiência do uso da água do Cinturão Econômico do Rio Yangtze" (DING et al., 2019). “Há um reconhecimento entre os gestores de que a gestão sustentável de bacias hidrográficas precisa não só levar em conta as diversas formas como os seres humanos se beneficiam do ambiente, mas também incorporar o impacto das ações humanas no sistema natural" (KHAN et al., 2017). Estas duas classes tratam do sistema de gerenciamento das águas, tanto para ambientes públicos quanto em ambientes empresariais que se utilizam de água como seu principal recurso.

\section{Classe 3: Uso Agrícola, Industrial e doméstico da água}

Pertencendo ao terceiro eixo, a terceira classe aborda os textos relacionados ao uso agrícola, industrial e doméstico da água e seus padrões de qualidade para o uso, esse tema contempla 13,84\% de todos os STs presentes nos artigos pesquisados, alguns dos textos afirmam: "Há muitos motivos de preocupação sobre a qualidade da água para uso doméstico" (JUSTES et al., 2014); “Para tornar mais grave a situação, a variabilidade e as mudanças climáticas reduzem o abastecimento de água disponível para usos domésticos e produtivos" (GABRIELSSON et al., 2013). 
Diversos materiais analisados nesta pesquisa, tratam sobre os desafios e as perspectivas da elaboração de políticas e diretrizes quanto ao uso consciente e a disponibilidade constante da água em ambientes que apresentam grandes aglomerações populacionais, a exemplo do que foi descrito por loris, (2012). Assim como a mediação de conflitos em áreas que detém pouco ou nenhum recursos hídrico disponível, como ocorre nos países que dependem das bacias hidrográficas na África Subsaariana e África do sul, como relatado nos trabalhos de Komakech (2012) e Siebrits (2014). Exemplos de boas práticas de governança são abordados em alguns trabalhos, já que a crise da água pode se relacionar com as falhas de governança e não de disponibilidade (WALKER, 2014).

Os recursos hídricos são descritos ao longo do tempo como recursos superalocados para o uso na agricultura, e a possibilidade de uma contenção extrema da água potável pode trazer prejuízos à segurança alimentar do mundo (SILVEIRA et al., 2019; VAN DIJK et al., 2013). O uso da água em ambientes agrícolas é contemplado neste eixo, já que a produção agrícola ainda é a atividade de maior uso da água potável do mundo, dentre os múltiplos usos, sendo por isso, de fundamental importância a adoção de mecanismos que beneficiem o aumento da eficiência dos recursos disponíveis, sem que a produtividade das culturas seja afetada (SINGH et al., 2016). Alguns trabalhos indicaram que a irrigação informal é generalizada nas zonas agrícola de pouco acesso, em uma grande diversidade de ambientes, caracterizada por uma diversidade igualmente grande de usos locais e estratégias de uso da água (GUTIERREZ-MALAXECHEBARRIA, 2013; SINGH et al., 2016).

Neste sentido é importante observar a necessidade do desenvolvimento das políticas públicas que enfoquem a promoção de melhores práticas agrícolas, e o desenvolvimento de tecnologias que promovam economia hídrica rural e a melhoria da produção agrícola. Muitos trabalhos demostram que o uso informal e indiscriminado da água em ambientes rurais ainda é presente no mundo, principalmente nos locais de pouco acesso à assistência rural.

Foi possível observar que existem muitas publicações científicas que tratam sobre a valorização econômica da água para uso doméstico em detrimento do uso da água na indústria, sugerindo que o desenvolvimento de inovações que tratam do tratamento de águas residuais domésticas devem ter maior prioridade, de modo que haja uma distinção clara entre indústria e consumidor residencial, uma vez que as indústrias deterioram este recurso com maior velocidade e em maior volume, Gamze-Tolun et al. (2012).

\section{Classe 4: Infraestrutura de distribuição e coleta sustentável da água}

A classe quatro mostrou ser a classe mais afastada das demais que estão no segundo eixo, dela surgem artigos que tratam sobre infraestrutura e distribuição da água, assim como coleta de água residual. Compreende $25,89 \%$ dos STs presentes no corpus da pesquisa em sua maioria publicados no ano de 2014 . Fragmentos dos principais artigos analisados ilustram seu conteúdo: "A construção da Sponge City é uma abordagem eficaz para resolver os problemas de água urbana, particularmente para o Waterlogging" (XIA et al., 2017); “Embora a reciclagem de água potável possa aumentar um abastecimento urbano de água de outro modo limitado, a ameaça da oposição pública ou política muitas vezes paira sobre si mesma" 
(ORMEROD et al., 2017).

Outros trabalhos observados nesta pesquisa sugerem que o eixo também trata sobre as capacidades de carga de recursos hídricos utilizando para isso modelagens teóricas, como por exemplo a base metabólica sobre os recursos hídricos regionais dos corpos hídricos utilizados em ambientes de grande aglomeração. Esse método leva em conta um sistema de índice de avaliação que considera as características dos recursos hídricos regionais, o sistema socioeconômico e o princípio do desenvolvimento sustentável (REN et al., 2016).

Tal eixo descreve bem a preocupação dos pesquisadores quanto ao aspecto de economia hídrica relacionada ao uso consciente nos ambientes urbanos, relacionando tais medidas aos posicionamentos políticos da comunidade envolvida. Próxima às classes pertencentes no segundo eixo, esta classe apresenta os trabalhos relacionados à gestão pública no meio urbano em toda cadeia de distribuição e coleta. Tais propostas buscam refletir a intensidade do consumo de água e a eficiência da produção.

As quatro classes que pertencem ao segundo eixo circulam na esfera dos poderes públicos e privados sobre as melhores formas de governança de suas fontes hídricas e distribuição de forma consciente e sustentável, desde o uso industrial e urbano, culminando em uma boa gestão de coleta de águas residuais capazes de serem reutilizadas. Isto demonstra que a necessidade de inovação vai muito além do desenvolvimento industrial, em que reside a necessidade de melhores formas de governança, melhores formas de outorga e cobrança, assim como ações mais efetivas para o uso sustentável do poder público no geral (ALMEIDA et al., 2016).

\section{Classe 5: Reservas subterrâneas}

Por fim a classe cinco se mostra mais distante das demais e trata dos ambientes subterrâneos de água (aquíferos) e seus segmentos. Ele contempla 16,96\% do total dos STs publicados, e os anos mais frequentes de publicação são 2017 e 2019. Pode ser descrito pelos seguintes UCls elementares: "Simulação numérica da variação estrutural do efeito do aquífero na circulação regional de águas subterrâneas", (DAl et al., 2017); “Operadores realizaram estudos geoquímicos e isotópicos locais sobre águas subterrâneas para estabelecer características de base para melhorar a compreensão da conectividade das formações de abastecimento", (BIRKS et al., 2019).

Textos que tratam desta temática circundam sobre as técnicas que contemplam formas de compreender o fluxo de águas subterrâneas em diferentes localidades, assim como a manutenção destes corpos d’água tão importantes para a humanidade. Tem-se como exemplo o trabalho proposto por Birks et al. (2019), que aborda as análises geoquímicas e de isótopo em águas subterrâneas para estabelecer características de base, de maneira a melhorar a compreensão da conectividade do abastecimento e eliminação de águas neste tipo de ambiente, assim como trabalhos que averiguam os impactos estruturais causados nestes ambientes devido à atividade da prospecção (DAl et al., 2017).

\section{Patentometria}

Dos textos patentários recuperados, surgiram 1992 STs, 7945 UCls, 404684 ocorrências, 9578 
palavras distintas e 3834 hapax. Exibindo uma frequência média de ocorrência de 0,95\% por palavra e uma frequência média de 40,03\% de ocorrência por ST. O resultado de CHD apresentou 7945 CDE, aproveitando desse total 6865, representando, assim, um aproveitamento de $86,41 \%$ do corpus estudado (Figura 4). Deles resultaram também quatro classes e quatro eixos. Os textos patentários, por serem naturalmente mais técnicos e descritivos, apresentaram neste estudo palavras representadas por substantivos e adjetivos.

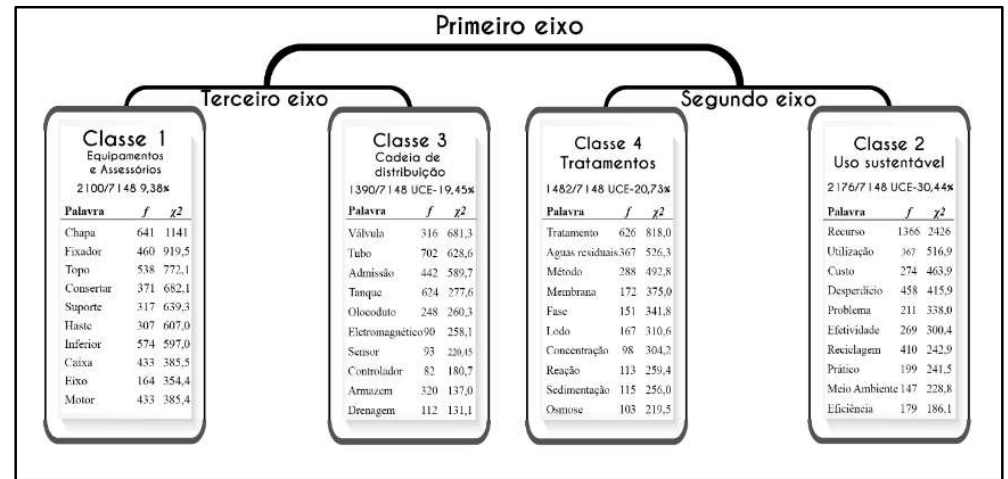

Figura 4: Representação das classes formadas pelas principais palavras observadas nos textos patentários.

O primeiro eixo se desmembra em mais dois eixos: o terceiro eixo contendo duas classes, a classe 3 que trata de temas relacionados à cadeia de distribuição, ao acesso à água e à recuperação de esgoto, e a classe 1 que trata de acessórios utilizados na logística; e o segundo eixo que também apresenta duas classes, a classe 4 que aborda sobre tratamento de água potável e a classe 2 que trata sobre reuso sustentável da água.

$\mathrm{Na}$ primeira classe que trata sobre equipamentos acessórios e equipamentos de distribuição, destaca-se como maior depositante a Chongqing Water Resources \& Electric College. A Faculdade Chongqing de Tecnologia Verde e Inteligente é uma instituição pública vinculada à Academia Chinesa de Ciências, ao Escritório Estadual das Três Gargantas e ao Município de Chongqing do Governo Popular da China. A Instituição desenvolve pesquisa em diversas áreas relacionadas à produção de energia e distribuição hídrica sustentável e verde (CAS, 2020). A patente que mais descreve esta classe é a CN208899580, "A invenção descreve um novo dispositivo de proteção dupla para hidrante externo" (ZHENG, 2018).

A segunda classe observada apresenta patentes que tratam sobre equipamentos desenvolvidos para o uso sustentável da água. O depositante que apresentou maior quantidade de registros patentários foi a Guangdong Poktai Sanitary Ware Tech, empresa chinesa especializada em venda de bidê inteligente e demais acessórios. A patente que melhor descreve este é a CN109025082 (MA, 2018), "Edifício de energia solar verde com dispositivo de economia de água", assim como a patente CN110205995, "Dispositivo e método de processamento de reutilização de água da cauda da usina" (MA et al., 2019).

A terceira classe trata sobre cadeia de distribuição e acesso a água e recuperação de esgoto doméstico. A principal depositante observada foi a Universidade North China Water Resources \& Electric Power, assim como a Chongqing Water Resources \& Electric College, que é um instituto de ensino e pesquisas tecnologias, sediada na República da China. Tendo como patente descritiva desta classe foi observada a, patente de registro CN109205783 “Método para processamento de esgoto doméstico a baixa temperatura 
para remoção simultânea de carbono, nitrogênio e fósforo com base no processo A SBR" (LIU et al., 2019).

\section{Textos patentários por Classificação Internacional de Patentes IPC}

A IPC é o sistema de classificação internacional adotado em mais de 100 países atualmente, foi criada a partir do Acordo de Estrasburgo (1971), cujas áreas tecnológicas são divididas nas classes A a H. Para cada classe há subclasses, grupos principais e grupos, através de um sistema hierárquico (INPI, 2020).

Dos principais códigos IPCs utilizados na busca inicial dos trabalhos, surgiram mais seis sessões vinculadas às patentes depositadas, conforme visto na Figura 5. Observa-se que o maior número de patentes é representado pela Classificação Internacional de Patentes (CIP) ' $C$ ', representando compostos químicos e equipamentos metalúrgicos, e a subseção 'C02' que trata de tratamento de água, de águas residuais, de esgotos ou de lamas e lodos (WIPO, 1970).

A segunda maior seção de ocorrência foi a 'E', Construções fixas, com 934 patentes. É salutar ressaltar que a subseção de maior ocorrência deste grupo foi a 'E03' que trata de abastecimento de água e sistemas de esgoto, no total de 899 Patentes. Tais resultados refletem significativamente as principais classes observadas nesta pesquisa. Segundo o CHD as maiores classes observadas foram a classe 2, que trata do uso sustentável da água e a classe 4 que trata sobre tratamento da água e lodo. Da mesma forma, os clusters por IPC demostraram haver tendência similar do volume por agrupamento que os principais clusters formados pela CHD, mostrando que, as patentes observadas neste estudo apresentaram uma maior preocupação com o uso sustentável e com o tratamento da água.

As demais preocupações presentes nas duas outras classes, tratam sobre transporte, distribuição e acesso à água (classe 3), por estarem associadas às patentes descritas como pertencentes ao IPC 'B' (performance operação e transporte) e à sessão 'H' (Necessidades humanas).

A principal subseção observada foi a 'E03' (Abastecimento de água; Rede de esgotos). Esta subseção pertence às patentes que tratam de instalações ou métodos para obter, coletar ou distribuir água, encanamentos de água potável, privadas, esgotos e foças (WIPO, 2018). Os demais textos patentários podem ser incluídos na classe 1 da CHD, que trata de equipamentos assessórios na cadeia da gestão dos recursos hídricos, as sessões 'G', 'D' e 'H' do IPC descreve bem estes conteúdos.

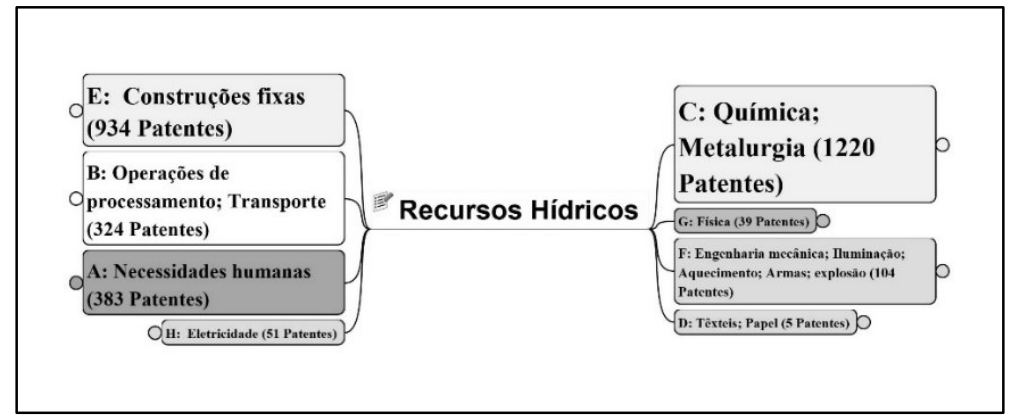

Figura 5: Principais Seções por Classificação Internacional de Patentes IPC dos textos patentários relacionados a recursos hídricos.

Por meio do uso desta metodologia, os textos patentários relacionados às áreas de recursos hídricos exibiram quatro classes distintas, e segmentadas em dois eixos paralelos, sendo elas: 
equipamentos/assessórios, uso sustentável, cadeia de distribuição e tratamentos.

Os resultados da presente abordagem sistemática para revisão de artigos de pesquisa e textos patentários demonstram haver cinco áreas de desenvolvimento de tecnologias direcionadas aos recursos hídricos, quando levantados materiais entre os anos de 2010 a 2020. Pode-se assim evidenciar a importância de entender essas áreas de estudos nos mais diversos contextos de uso e gerenciamento dos recursos hídricos.

Tendo em vista os aspectos apresentados, percebe-se que a maioria dos artigos analisados tratam sobre a classe temática governança pública e inovação em recursos hídricos, observando-se uma direta relação deste tema com os demais resultantes. Com a abordagem aqui estudada foi possível observar que dos artigos científicos observados obteve-se como resultado cinco clusters, dos quais quatro são diretamente relacionados e um indiretamente.

A partir da aplicação do conjunto de metodologias e com base nos resultados obtidos foi possível concluir que a proposta desenvolvida se mostra viável para ser aplicada em casos em que se pretenda verificar as principais áreas de estudo das temáticas vinculadas aos Recursos Hídricos.

\section{CONCLUSÕES}

Este estudo demonstra que a abordagem prospectiva proposta como ferramenta foi eficaz na extração, avaliação, organização e disponibilização de dados sobre pesquisa científica assim como tecnologias patenteadas relacionadas aos recursos hídricos. Como todas as ferramentas de busca, classificação, análise, estatística e categorização utilizadas nesta abordagem são disponibilizadas gratuitamente, esta pesquisa pode ajudar pesquisadores, inventores e instituições de ensino e pesquisa a alcançarem novos níveis de desenvolvimento científico e tecnológico.

Os dados resultantes deste teste podem ser facilmente replicados. A abordagem descrita aqui pode ser usada para avaliar publicações de patentes sobre qualquer assunto, de acordo com o interesse de cada pesquisador, estudante ou empresa. Embora este trabalho seja capaz de descrever minuciosamente a conjuntura das pesquisas e atividades inventivas sobre recursos hídricos, são recomendadas futuras pesquisas em outras áreas, a fim de que sejam acuradas todas as fases e ferramentas utilizadas nesta abordagem.

Devido aos seus critérios de inclusão precisos e ao amplo escopo da literatura que trata sobre recursos hídricos, esta abordagem pode ter excluído inadvertidamente alguns textos que discutem fatos relevantes sobre a inovação para a área proposta. Para a obtenção de resultados com maior precisão, pesquisas futuras devem rastrear artigos e textos patentários depositados em diferentes bancos de dados e examiná-los do mesmo modo que o utilizado nesta pesquisa, nesse sentido, as limitações de conteúdos de estudo devem ser exploradas em estudos adicionais.

\section{REFERÊNCIAS}

ALMEIDA, M. A.; CURI, W. F.. Gestão do uso de água na bacia do Rio Paraíba, PB, Brasil com base em modelos de outorga e cobrança. Revista Ambiente \& Água, v.11, n.4, p.9891005, 2016. 
ANA. Agência Nacional de Águas. Água subterrânea. ANA, 2020.

ANA. Agência Nacional de Águas. Atlas Irrigação. ANA, 2019.

BASTIAN, M.; HEYMANN, S.; JACOMY, M.. Gephi: An Open Source Software for Exploring and Manipulating Networks. In: THIRD INTERNATIONAL AAAI CONFERENCE ON WEBLOGS AND SOCIAL MEDIA, 19. Anais. 2009.

BIRKS, S. J.; FENNELL, J. W.; GIBSON, J. J.; YI, Y.; MONCUR, M. C.; BREWSTER, M.. Using regional datasets of isotope geochemistry to resolve complex groundwater flow and formation connectivity in northeastern Alberta, Canada. Applied Geochemistry, v.101, p.140-159, 2019. DOI: https://doi.org/10.1016/j.apgeochem.2018.12.013

CAMARGO, B. V.; JUSTO, A. M.. IRAMUTEQ: um software gratuito para análise de dados textuais. Temas em Psicologia, v.21, n.2, p.513-518, 2013.

CHANAPATHI, T.; THATIKONDA, S.; KEESARA, V. R.; PONGURU, N. S.. Assessment of water resources and crop yield under future climate scenarios: A case study in a Warangal district of Telangana, India. Journal of Earth System Science, v.129, n.1, p.20, 2019. DOI: https://doi.org/10.1007/s12040-019-1294-3

CAS. Chinese Academy of Sciences. Chongqing Institute of Green and Intelligent Technology, Chinese Academy of Sciences. 2020.

CONVERY, F. J.. Reflections-shaping water policy: What does economics have to offer?. Review of Environmental Economics and Policy, v.7, n.1, p.156-174, 2013. DOI: https://doi.org/10.1093/reep/res017

COSTA, A. B.; ZOLTOWSKI, A. P. C.; KOLLER, S. H.; TEIXEIRA, M. A. P.. Construção de uma escala para avaliar a qualidade metodológica de revisões sistemáticas. Ciência \& Saúde Coletiva, v.20, n.8, p.2441-2452, 2015. DOI: https://doi.org/10.1590/1413-81232015208.10762014

DAI, F.-G.; ZHANG, F.-W.; WANG, B.; YAO, H.-C.; CHEN, L.. Numerical simulation of structural variation of aquifer effect on regional groundwater circulation. Acta Geoscientica Sinica, v.38, p.64-68, 2017. DOI: https://doi.org/10.3975/cagsb.2017.s1.17

DEL CORSO, J.-P.; KEPHALIACOS, C.; PLUMECOCQ, G.. Legitimizing farmers' new knowledge, learning and practices through communicative action: Application of an agroenvironmental policy. Ecological Economics, v.117, p.86-96, 2015. DOI: https://doi.org/10.1016/j.ecolecon.2015.05.017

DING, X.; TANG, N.; HE, J.. The threshold effect of environmental regulation, FDI agglomeration, and water utilization efficiency under "double control actions"-An empirical test based on Yangtze River Economic Belt. Water, v.11, n.3, 2019. DOI: http://doi.org/10.3390/w11030452

DJEBOU, D. C. S.. Bridging drought and climate aridity. Journal of Arid Environments, v.144, p.170-180, 2017. DOI: https://doi.org/10.1016/i.jaridenv.2017.05.002
EXTERCKOTER, R. K.; SCHLINDWEIN, S. L.. Aplicabilidade do Índice de Qualidade do Uso da Água (IQUA) como ferramenta de auxílio para a gestão de bacias hidrográficas. Geosul, v.29, n.58, p.7-28, 2015.

FERNANDES, B.. Manual Iramuteq. 2016.

FERNANDES, J. S. G.; ANDRADE, M. S.. Revisão sobre a doença de alzheimer: diagnóstico, evolução e cuidados. Psicologia, Saúde \& Doenças, v.18, n.1, p.131-140, 2017.

FERRAZ, R. R. N.; QUONIAM, L.; REYMOND, D.; MACCARI, E. A.. Example of open-source OPS (Open Patent Services) for patent education and information using the computational tool Patent2Net. World Patent Information, v.46, p.21-31, 2016.

GABRIELSSON, S.; RAMASAR, V.. Widows: Agents of change in a climate of water uncertainty. Journal of Cleaner Production, v.60, p.34-42, 2013. DOI: https://doi.org/10.1016/i.jclepro.2012.01.034

GAMZE-TOLUN, L.; ERGENEKON, S.; MURAT HOCAOGLU, S.; SUHA DONERTAS, A.; COKACAR, T.; HUSREVOGLU, S.; POLAT BEKEN, C.; BABAN, A.. Socioeconomic Response to Water Quality: a First Experience in Science and Policy Integration for the Izmit Bay Coastal System. Ecology and Society, v.17, n.3, 2012. DOI: http://doi.org/10.5751/ES-04984-170340

GAVASSO, M. S. B.; FERNANDES, J. S. G.; ANDRADE, M. S.. Revisão sistemática de estudos sobre habilidades sociais: avaliação e treinamento. Ciências \& Cognição, v.21, n.1, 2016.

GÜÇLÜ, Y. S.. Multiple Şen-innovative trend analyses and partial Mann-Kendall test. Journal of Hydrology, v.566, p.685-704, 2018. DOI: https://doi.org/10.1016/i.jhydrol.2018.09.034

GUTIERREZ-MALAXECHEBARRIA, A.-M.. Informal irrigation in the colombian andes: Local practices, national agendas, and options for innovation. Mountain Research and Development, v.33, n.3, p.260-268, 2013. DOI: https://doi.org/10.1659/MRD-JOURNAL-D-12-00116.1

HASAN, I.; KHALIL, F.; SUN, X.. The Impacts of Intellectual Property Rights Protection on Cross-Border M\&As. Quarterly Journal of Finance, v.07, n.03, p.1750005, 2017. DOI: https://doi.org/10.1142/S2010139217500057

HOOCK, C.; BROWN, A.. Early Certainty in patent cases involving by opposition proceedings. World Patent Information, v.61, p.101948, 2020. DOI: https://doi.org/10.1016/j.wpi.2020.101948

HU, Q.; YANG, Y.; HAN, S.; YANG, Y.; AI, Z.; WANG, J.; MA, F.. Identifying changes in irrigation return flow with gradually intensified water-saving technology using HYDRUS for regional water resources management. Agricultural Water Management, v.194, p.33-47, 2017. DOI: https://doi.org/10.1016/j.agwat.2017.08.023

INPI. Instituto Nacional da Propriedade Industrial. Classificação de patentes. INPI, 2020.

INPI. Instituto Nacional da Propriedade Industrial. Diretrizes de exame de pedido de patente. INPI, 2011. 
IORIS, A. A. R.. The Political Geography of Environmental Regulation: Implementing the Water Framework Directive in the Douro River Basin, Portugal. Scottish Geographical Journal, v.128, n.1, p.1-23, 2012. DOI: https://doi.org/10.1080/14702541.2012.676667

IWANAGA, T.; PARTINGTON, D.; TICEHURST, J.; CROKE, B. F. W.; JAKEMAN, A. J.. A socio-environmental model for exploring sustainable water management futures: Participatory and collaborative modelling in the Lower Campaspe catchment. Journal of Hydrology: Regional Studies, v.28, p.100669, 2020. DOI: https://doi.org/10.1016/j.ejrh.2020.100669 JUSTES, A.; BARBERÁN, R.; FARIZO, B. A.. Economic valuation of domestic water uses. Science of the Total Environment, v.472, p.712-718, 2014. DOI: https://doi.org/10.1016/i.scitotenv.2013.11.113

KHAN, H. F.; YANG, Y. C. E.; XIE, H.; RINGLER, C.. A coupled modeling framework for sustainable watershed management in transboundary river basins. Hydrology and Earth System Sciences, v.21, n.12, p.6275-6288, 2017. DOI: https://doi.org/10.5194/hess-21-6275-2017

KITCHENHAM, B.. Procedures for performing systematic reviews. Keele University, v.33, n.2004, p.1-26, 2004.

KITCHENHAM, B.; BRERETON, O. P.; BUDGEN, D.; TURNER, M.; JOHN BAILEY; LINKMAN, S.. Systematic literature reviews in software engineering: A systematic literature review. Information and Software Technology, v.51, n.1, p.7-15, 2009. DOI: https://doi.org/10.1016/j.infsof.2008.09.009

KOMAKECH, H. C.; VAN DER ZAAG, P.; MUL, M. L.; MWAKALUKWA, T. A.; KEMERINK, J. S.. Formalization of water allocation systems and impacts on local practices in the Hingilili sub-catchment, Tanzania. International Journal of River Basin Management, v.10, n.3, p.213-227, 2012. DOI: https://doi.org/10.1080/15715124.2012.664774

LAPES, L. P. E. S.. StArt - LaPES: Laboratório de Pesquisa em Engenharia de Software. 2005.

LEITE, J. L.; LEITE, J. L.. Publicar ou perecer: a esfinge do produtivismo acadêmico. Revista Katálysis, v.20, n.2, p. 207215, ago. 2017. DOI: https://doi.org/10.1590/198202592017v20n2p207

\section{LIU, S.; FU, J.; LIU, B.; ZHAO, W.. Método para} processamento de esgoto doméstico a baixa temperatura para remoção simultânea de carbono, nitrogênio e fósforo com base no processo A-SBR. 2019.

MA, J.. Edifício de energia solar verde com dispositivo de economia de água. 2018.

MA, J.; SHI, C.. Power plant tail water reuse processing device and method. 2019.

MARCHAND, P.; RATINAUD, P.. L'analyse de similitude appliquée aux corpus textuels: les primaires socialistes pour l'élection présidentielle française (septembre-octobre 2011). Actes des 11eme Journées internationales d'Analyse statistique des Données Textuelles. JADT, v.2012, p. 687699, 2012.
MARQUES, J. P. F. R.; MARQUES, R. F. R.. A natureza e o licere de pedido de patente de invenção no Brasil: Concorrência desleal e direito da concorrência em matéria de medicamentos genéricos perante (ameaça de) ajuizamento de ação de infração por parte do requerente de pedido de patente de medicamento de referência. Revista em Propriedade Intelectual Direito Contemporâneo, v.11, n.2, p.1-61, 2017.

MELO, M. G. G.; MEDEIROS, R. S.; SAMPAIO, P. T. B.; VIEIRA, G.. Sustainability issues: Riparian vegetation and its importance in the hydrological cycle in Amazonian ecosystems. Journal of Security and Sustainability ,v.7, n.4, p.861-868, 2018. DOI: https://doi.org/10.9770/jssi.2018.7.4(21)

MONTEIRO, A.; MONTEIRO, A.. O que é a Inovação Social? Maleabilidade Conceitual e Implicações Práticas. Dados, v.62, n.3, 2019. DOI:

http://doi.org/10.1590/001152582019187

NASCIMENTO, A. R. A.; MENANDRO, P. R. M.. Análise lexical e análise de conteúdo: uma proposta de utilização conjugada. Estudos e Pesquisas em Psicologia, v.6, n.2, p.72-88, 2006

OCTAVIANO, F. R.; FELIZARDO, K. R.; MALDONADO, J. C.; FABBRI, S. C. P. F.. Semi-automatic selection of primary studies in systematic literature reviews: is it reasonable? Empirical Software Engineering, v.20, n.6, p.1898-1917, 2015. DOI: https://doi.org/10.1007/s10664-014-9342-8

ORMEROD, K. J.; SILVIA, L.. Newspaper coverage of potable water recycling at Orange County water district's groundwater replenishment system, 2000-2016. Water, v.9, n.12, 2017. DOI: http://doi.org/10.3390/w9120984

PIRES, W. J. M.. As disputas pela água e o papel dos aquíferos do Brasil no cenário internacional do século XXI. 2018.

REN, C.; GUO, P.; LI, M.; LI, R.. An innovative method for water resources carrying capacity research - Metabolic theory of regional water resources. Journal of Environmental Management, v.167, p. 139-146, 2016. DOI: https://doi.org/10.1016/j.jenvman.2015.11.033

SHEA, B. J.; GRIMSHAW, J. M.; WELLS, G. A.; BOERS, M.; ANDERSSON, N.; HAMEL, C.; PORTER, A. C.; TUGWELL, P.; MOHER, D.; BOUTER, L. M.. Development of AMSTAR: a measurement tool to assess the methodological quality of systematic reviews. BMC Medical Research Methodology, v.7, n.1, p.10, 2007. DOI: https://doi.org/10.1186/1471$\underline{2288-7-10}$

SIDHU, B. S.; KANDLIKAR, M.; RAMANKUTTY, N.. Power tariffs for groundwater irrigation in India: A comparative analysis of the environmental, equity, and economic tradeoffs. World Development, v.128, p.104836, 2020.

SIEBRITS, R. M.; WINTER, K.; BARNES, J.; DENT, M. C.; EKAMA, G.; GINSTER, M.; HARRISON, J.; JACKSON, B.; JACOBS, I.; JORDAAN, A.; KASAN, H. C.; KLOPPERS, W.; LE ROUX, R.; MAREE, J.; MOMBA, M. N. B.; MUNNIK, A. V.; O'KEEFFE, J.; SCHULZE, R.; SILBERBAUER, M.; VAN ZYL, J. E.. Priority water research questions for South Africa developed 
through participatory processes. Water SA, v.40, n.2, p.199209, 2014. DOI: https://doi.org/10.4314/wsa.v40i2.2

SILVEIRA, R. N. C. M.; PEIXOTO, F. D. S.; COSTA, R. N. T.; CAVALCANTE, I. N.. Efeitos da Seca em Perímetros Irrigados no Semiárido Brasileiro. Anuário do Instituto de Geociências, v.41, n.2, p.268-275, 2019.

SINGH, S.; SRIVASTAVA, P.; MITRA, S.; ABEBE, A.. Climate variability and irrigation impacts on streamflows in a Karst watershed: A systematic evaluation. Journal of Hydrology: Regional Studies, v.8, p.274-286, 2016. DOI: https://doi.org/10.1016/j.ejrh.2016.07.001

SLAMET, C.; RAHMAN, A.; RAMDHANI, M. A.; DARMALAKSANA, W.. Clustering the verses of the Holy Qur'an using K-means algorithm. Asian Journal of Information Technology, v.15, n.24, p.5159-5162, 2016.

SOUZA, M. A. R.; WALL, M. L.; THULER, A. C. M. C.; LOWEN, I. M. V.; PERES, A. M.. O uso do software IRAMUTEQ na análise de dados em pesquisas qualitativas. Revista da Escola de Enfermagem da USP, v.52, 2018. DOI:

http://doi.org/10.1590/s1980-220×2017015003353

TERINK, W.; HURKMANS, R. T. W. L.; TORFS, P. J. J. F.; UIJLENHOET, R.. Evaluation of a bias correction method applied to downscaled precipitation and temperature reanalysis data for the Rhine basin. Hydrology and Earth System Sciences, v.14, n.4, p.687-703, 2010. DOI: https://doi.org/10.5194/hess-14-687-2010

TONELLI, M. J.; TONELLI, M. J.. Sobre Publicar. Revista de Administração de Empresas, v.56, n.6, p.594-594, 2016. DOI: https://doi.org/10.1590/s0034-759020160601

VAN DIJK, A. I.; BECK, H. E.; CROSBIE, R. S.; DE JEU, R. A.; LIU, Y. Y.; PODGER, G. M.; TIMBAL, B.; VINEY, N. R.. The Millennium Drought in southeast Australia (2001-2009): Natural and human causes and implications for water resources, ecosystems, economy, and society. Water Resources Research, v.49, n.2, p.1040-1057, 2013.
WALKER, G.. Water scarcity in England and Wales as a failure of (meta)governance. Water Alternatives, v.7, n.2, p.388413, 2014.

WANG, Y.; WU, X.; TORTAJADA, C.. Innovative and transformative water policy and management in China. International Journal of Water Resources Development, 2020. DOI: http://doi.org/10.1080/07900627.2020.1716694

WANG, Y.; XU, Y.; TABARI, H.; WANG, J.; WANG, Q.; SONG, S.; HU, Z.. Innovative trend analysis of annual and seasonal rainfall in the Yangtze River Delta, eastern China.

Atmospheric Research, v.231, 2020. DOI http://doi.org/10.1016/i.atmosres.2019.104673

WIPO. World Intellectual Proper. Patent cooperation treaty yearly review. Place of publication not identified. WIPO, 2018.

WIPO. World Intellectual Proper. Tratado de Cooperação em matéria de Patentes. WIPO, 1970.

WOLFFENBUTTEL, R. F.; WOLFFENBUTTEL, R. F.. Inovação econômica: trajetórias e contribuições de uma agenda de pesquisa sociológica. Sociologias, v.20, n.48, p.310-323, 2018. DOI: https://doi.org/10.1590/15174522-020004832

XIA, J.; ZHANG, Y. Y.; XIONG, L. H.; HE, S.; WANG, L. F.; YU, Z. B.. Opportunities and challenges of the Sponge City construction related to urban water issues in China. Science China Earth Sciences, v.60, n.4, p.652-658, 2017. DOI: https://doi.org/10.1007/s11430-016-0111-8

XIE, X.; JIANG, X.; ZHANG, T.; HUANG, Z.. Study on impact of electricity production on regional water resource in China by water footprint. Renewable Energy, v.152, p.165-178, 2020. DOI: https://doi.org/10.1016/i.renene.2020.01.025

ZHENG, C.. The invention discloses a novel outdoor fire hydrant double-protection device. 2018.

A CBPC - Companhia Brasileira de Produção Científica (CNPJ: 11.221.422/0001-03) detém os direitos materiais desta publicação. Os direitos referem-se à publicação do trabalho em qualquer parte do mundo, incluindo os direitos às renovações, expansões e disseminações da contribuição, bem como outros direitos subsidiários. Todos os trabalhos publicados eletronicamente poderão posteriormente ser publicados em coletâneas impressas sob coordenação da Sustenere Publishing, da Companhia Brasileira de Produção Científica e seus parceiros autorizados. Os (as) autores (as) preservam os direitos autorais, mas não têm permissão para a publicação da contribuição em outro meio, impresso ou digital, em português ou em tradução. 